Lymphoma

\title{
CXCR4 hyperactivation cooperates with TCL1 in CLL development and aggressiveness
}

\author{
Richard Lewis $\mathbb{D}^{1,2}$, H. Carlo Maurer ${ }^{3}$, Nikita Singh ${ }^{1}$, Irene Gonzalez-Menendez ${ }^{4}$, Matthias Wirth (D) ${ }^{1}$, Markus Schick ${ }^{1}$, Le $^{2}$ Zhang ${ }^{1}$, \\ Konstandina Isaakidis ${ }^{1}$, Anna Katharina Scherger ${ }^{2}$, Veronika Schulze ${ }^{1}$, Junyan Lu ${ }^{5}$, Thorsten Zenz (D $^{6}$, Katja Steiger (DD ${ }^{7}$, Roland Rad ${ }^{8,9,10}$ \\ Leticia Quintanilla-Martinez ${ }^{4}$, Marion Espeli ${ }^{11,12,13}$, Karl Balabanian (iD ${ }^{11,12,13}$, Ulrich Keller (iD) 10,14,16凶 and Stefan Habringer (iD ${ }^{1,15,16 \bowtie}$
}

(c) The Author(s) 2021

\begin{abstract}
Aberrant CXCR4 activity has been implicated in lymphoma pathogenesis, disease progression, and resistance to therapies. Using a mouse model with a gain-of-function CXCR4 mutation $\left(C X C R 4^{C 1013 G}\right)$ that hyperactivates CXCR4 signaling, we identified CXCR4 as a crucial activator of multiple key oncogenic pathways. CXCR4 hyperactivation resulted in an expansion of transitional B1 lymphocytes, which represent the precursors of chronic lymphocytic leukemia (CLL). Indeed, CXCR4 hyperactivation led to a significant acceleration of disease onset and a more aggressive phenotype in the murine E $\mu$-TCL1 CLL model. Hyperactivated CXCR4 signaling cooperated with TCL1 to cause a distinct oncogenic transcriptional program in B cells, characterized by PLK1/ FOXM1-associated pathways. In accordance, E $\mu$-TCL1;CXCR4 ${ }^{C 1013 G}$ B cells enriched a transcriptional signature from patients with Richter's syndrome, an aggressive transformation of CLL. Notably, MYC activation in aggressive lymphoma was associated with increased CXCR4 expression. In line with this finding, additional hyperactive CXCR4 signaling in the E $\mu$-Myc mouse, a model of aggressive B-cell cancer, did not impact survival. In summary, we here identify CXCR4 hyperactivation as a co-driver of an aggressive lymphoma phenotype.
\end{abstract}

Leukemia (2021) 35:2895-2905; https://doi.org/10.1038/s41375-021-01376-1

\section{INTRODUCTION}

CXCR4 is a G-protein-coupled receptor regulating hematopoietic stem cell homeostasis, myelopoiesis, lymphopoiesis, and homing of immune cells toward its ligand $\mathrm{C}-\mathrm{X}-\mathrm{C}$ motif chemokine 12 (CXCL12) [1-3]. CXCL12 binding induces a multitude of G-proteindependent and -independent signaling pathways including $\mathrm{PI} 3 \mathrm{~K} /$ AKT, MAPK/ERK, and PLC/Ca ${ }^{2+}$ signaling [4]. CXCR4 is phosphorylated at the $\mathrm{C}$-terminus and rapidly internalized after binding of CXCL12 [5]. Truncating mutations affecting the C-terminus lead to increased activity of CXCR4 signaling in response to its ligand by impairing receptor desensitization and internalization without impairing receptor expression level [6-8], which has been modeled in mice [9].

$B$ cells in particular are highly dependent on the interaction of CXCR4 and its ligand CXCL12 at multiple stages during the germinal center reaction [10]. Molecularly targeted imaging studies have revealed enhanced CXCR4 expression in various B-cell non-Hodgkin lymphomas [11-13]. CXCR4 is of particular interest in chronic lymphocytic leukemia (CLL) and diffuse large B-cell lymphoma (DLBCL), having been associated with adverse prognosis in both diseases $[14,15]$. CXCR4 is overexpressed in CLL patients and involved in interactions of CLL cells with their microenvironment, specifically the protection from apoptosis by the provided ligand CXCL12 [16-18]. In DLBCL, CXCR4 expression correlates with bone marrow infiltration [19] and has been implied in mediating resistance to B-cell receptor and PI3K inhibitors [20].

Genetically engineered mouse models for CLL and aggressive B-cell lymphoma, specifically the $E \mu$-TCL1 mouse model of CLL [21] and the $E \mu-M y c$ mouse model of aggressive MYC-induced B-cell lymphoma [22], are essential for studying leukemia/lymphoma pathogenesis and complex biological systems like chemokine receptor pathways in B-cell pathobiology. The E $\mu$-TCL1 mouse, in

\footnotetext{
${ }^{1}$ Department of Hematology, Oncology and Cancer Immunology, Campus Benjamin Franklin, Charité - Universitätsmedizin Berlin, corporate member of Freie Universität Berlin and Humboldt-Universität zu Berlin, Berlin, Germany. ${ }^{2}$ School of Medicine, Technische Universität München, Munich, Germany. ${ }^{3}$ Internal Medicine II, School of Medicine, Technische Universität München, Munich, Germany. ${ }^{4}$ Institute of Pathology and Neuropathology and Comprehensive Cancer Center Tübingen, Eberhard Karls Universität Tübingen, Tübingen, Germany. ${ }^{5}$ European Molecular Biology Laboratory (EMBL), Heidelberg, Germany. ${ }^{6}$ Department of Medical Oncology and Hematology, Universitätsspital and Universität Zürich, Zurich, Switzerland. ${ }^{7}$ Institute of Pathology, Technische Universität München, Munich, Germany. ${ }^{8}$ TranslaTUM, Center for Translational Cancer Research, Technische Universität München, Munich, Germany. ${ }^{9}$ Institute of Molecular Oncology and Functional Genomics, TUM School of Medicine, Technische Universität München, Munich, Germany. ${ }^{10}$ German Cancer Consortium (DKTK), German Cancer Research Center (DKFZ), Heidelberg, Germany. ${ }^{11}$ Université de Paris, Institut de Recherche Saint-Louis, EMiLy, INSERM U1160, Paris, France. ${ }^{12}$ CNRS, GDR3697 "Microenvironment of Tumor Niches", Micronit, France. ${ }^{13}$ OPALE Carnot Institute, The Organization for Partnerships in Leukemia, Hôpital Saint-Louis, Paris, France. ${ }^{14}$ Max-Delbrück-Centrum für Molekulare Medizin, Berlin, Germany. ${ }^{15}$ Berlin Institute of Health at Charité (BIH), Berlin, Germany.




which B-cell-directed TCL1 expression drives development of a CLL-like disease, is the most commonly used model for high-risk CLL and has been extensively used for mechanistically assessing oncogenes and tumor suppressors in CLL [23, 24]. By using the $E \mu-T C L 1$ model, Chen et al. showed that downregulation of surface CXCR4 expression and inhibition of CXCR4 downstream signaling in CLL cells can be observed upon treatment with the clinically approved Bruton's tyrosine kinase (BTK) inhibitor ibrutinib [25]. CXCR4 inhibitors are currently under investigation in clinical trials in CLL [26].

Mutations of CXCR4 are present in DLBCL patients, but no functional studies have been performed to further inquire their function in this disease [27, 28]. Intriguingly, rare germline mutations in CXCR4 have been found in CLL patients with familial clustering [29] and mutations in regulatory regions of CXCR4 were discovered in biopsies of CLL with aggressive transformation (Richter's syndrome) [30]. It is however not resolved if and how they affect CXCR4 pathway activity and disease progression. Despite a plethora of evidence linking CXCR4 to the pathogenesis of CLL and DLBCL, it is unknown if and how enhancing CXCR4 pathway activity can alter the course of B-cell lymphoproliferation, and B-cell leukemia/ lymphoma development and progression.


models to interrogate the role of hyperactivated CXCR4 signaling in B-cell lymphoproliferation and B-cell leukemia/lymphoma pathogenesis. CXCR4 hyperactivation was achieved by a mouse model harboring CXCR4 ${ }^{C 1013 G}$, a mutation resulting in a truncated C-terminus missing phosphorylation sites for CXCR4 internalization and consequently enhanced downstream signaling [9]. This mutation is characteristic for WHIM-syndrome (warts, hypogammaglobulinemia, infections, myelokathexis) patients and allows the investigation of CXCR4 hyperactivation on B-cell lymphoproliferation and lymphoma development.

\section{MATERIAL AND METHODS \\ Animal experiments}

Genotyping was performed as previously described [9, 21, 31]. All analyses included heterozygous female and male animals on a C57BL/6J background. Animal caretakers, but not researchers performing experiments, were blinded for genotypes. Mice were allocated to groups based on genotype, thus no randomization was performed. All animal experiments were performed in accordance with Federation of European Laboratory Animal Science Associations guidelines and with permission of the respective authorities (Regierung von Oberbayern, Munich, Germany \& Landesamt für Gesundheit und Soziales, Berlin, Germany).

\section{Flow cytometry}

Staining was performed in PBS (ThermoFisher Scientific, Waltham, MA) containing $0.5 \%$ BSA (Carl Roth GmbH, Karlsruhe, Germany). To distinguish live from dead, cells were stained with $\mathrm{PI}, \mathrm{DAPI}$, or Invitrogen Fixable Aqua Dead Cell Stain Kit (ThermoFisher Scientific, Waltham, MA). Data analysis was done with FlowJo ${ }^{\text {TM }}$ Version 10.6.0 (FlowJo, Ashland, OR). An extensive list of all fluorescently labeled antibodies can be found in Supplementary Table T1. Gating strategies are depicted in Supplementary Fig. S1.

\section{Histopathological analysis}

Tissue samples were fixed in $4 \%$ formaldehyde for $48 \mathrm{~h}$, paraffin embedded, sectioned, and stained using H\&E. Samples of bone marrow were decalcified by incubating for 14 days with Osteosoft ${ }^{\circledR}$ (Sigma-Aldrich, Saint Louis, MI) after fixation.

\section{Immunohistochemistry}

Immunohistochemistry on murine tissues was performed on an automated immunostainer (Ventana Medical Systems Inc., Oro Valley, AZ) according to the company's protocols for open procedures with slight modifications. Appropriate positive and negative controls were used to confirm the adequacy of the staining. The histologic samples were analyzed by an experienced pathologist (L. Quintanilla-Martinez). Photomicrographic images were acquired with an Axioskop 2 plus Zeiss microscope equipped with a Jenoptik (Laser Optik System, Jena, Germany) ProgRes C10 plus camera and software. Objectives Plan-Neofluar used were as follows: 1.25/ $0,035,2.5 \times / 0.075,10 \times / 0.30,20 \times / 0.50$, and $40 \times / 0.75$. Final image preparation was performed with Adobe Photoshop CS6 (Adobe Inc., San José, CA).

\section{RNA sequencing}

B cells from spleen and bone marrow of WT, CXCR4 ${ }^{C 1013 G}, E \mu-T C L 1$, and $E \mu-T C L 1 ; C X C R 4^{C 1013 G}$ were isolated with CD19 directed magnetic beads (Miltenyi Biotec, Bergisch Gladbach, Germany). Only RNA samples with an RNA integrity number (RIN) $>7$ were used for RNA sequencing. RIN was determined using Agilent RNA 6000 Nano Kit (\# 5067-1513, Agilent, Santa Clara, CA) and the Agilent 2100 Expert software (version B.02.10.SI764). Library preparation and single-end sequencing was subsequently performed by Novogene (UK) (Cambridge, UK) on a HiSeq2500 (Illumina, San Diego, CA) with a sequencing depth of more than $20 \mathrm{M}$ reads/sample. Fastq files were subsequently mapped to the murine reference genome GRCm38 with STAR.

Reads and transcripts per million (TPM) were estimated for each transcript using the transcript sequences from the GENCODE Release 25 (GRCm38) and the Salmon software (v1.3.0). Counts and TPM were summarized at the gene level by summing up the transcript values for each corresponding gene. Bioinformatic analyses are described in the Supplementary methods section.

\section{Immunoblot analysis}

Protein extracts were prepared by incubating cell pellets in lysis buffer $(50$ $\mathrm{mM}$ Hepes, $150 \mathrm{mM} \mathrm{NaCl}, 1 \mathrm{mM}$ EDTA, $2.5 \mathrm{mM}$ EGTA and 0.1\% Tween) supplemented with $\mathrm{NaF}, \mathrm{PMSF}$, and $\mathrm{NaVO}_{4}$ followed by sonification. Protein lysates were fractioned on SDS PAGE gels, transferred to Immobilon-P (Millipore, Burlington, MA) membranes and incubated with specific antibodies, and developed with Chemostar PC ECL \& Fluorescence Imager (Intas Science Imaging, Göttingen, Germany). A list of antibodies used can be found in Supplementary Table T1. Quantification of protein expression levels was performed using ImageJ software (https://imagej.nih.gov/ij).

\section{Migration assay}

Splenocytes were resuspended in RPMI-medium with or without $1 \mu \mathrm{M}$ AMD3100 (Sigma-Aldrich, Saint Louis, MI). Cells were then loaded onto a Corning ${ }^{\circledR}$ Transwell ${ }^{\circledR}$ 96-well plate with $5 \mu \mathrm{m}$ pore size. (Corning Inc., Corning, NY) with the other side of the membrane containing medium with/without $50 \mathrm{nM}$ of CXCL12 (Peprotech, Rocky Hill, CT). Transwell ${ }^{\circledR}$ plates were incubated for $4 \mathrm{~h}$ at $37^{\circ} \mathrm{C}$. Migration of cells to the lower wells was measured using CountBright ${ }^{\mathrm{TM}}$ Absolute Counting Beads (ThermoFisher Scientific, Waltham, MA).

\section{Statistics}

Statistical analyses were performed using GraphPad Prism Version 9.0. (GraphPad Software, La Jolla, CA). Error bars represent standard deviation. Bar graphs represent the mean. In animal experiments, a single data point represents an individual mouse. No sample size calculations were performed. Data from at least three mice per group were reported. In survival analyses, mice were excluded when the cause of death was not transgene-related (e.g. fighting, birth complications). A two-tailed Student's $t$ test was used to compare quantitative data between 2 independent samples. When comparing three or more groups, a one-way ANOVA with Tukey correction for multiple comparisons was used to compare group means. Survival data were compared using a logrank (Mantel-Cox) test. Results with a $P$ value of less than 0.05 were considered significant.

\section{RESULTS}

CXCR4 hyperactivation promotes a distinct transcriptional signature in non-malignant $B$ cells

To investigate the consequences of hyperactivated CXCR4 on intrinsic signaling in B cells, we purified CD19+ B cells from CXCR4 $4^{+/ C 1013 G}$ mice (CXCR4 ${ }^{C 1013 G}$ from here on) and wild-type control mice (WT from here on) (Fig. 1a). To confirm enhanced CXCR4 signaling activity in CXCR4 ${ }^{C 1013 G} \mathrm{~B}$ cells, we performed immunoblotting for phosphorylation of the well-established downstream effector kinases ERK and AKT. As expected, both ERK and AKT phosphorylation were 


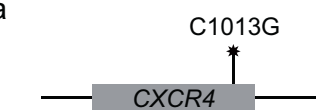

C-terminal truncating mutation

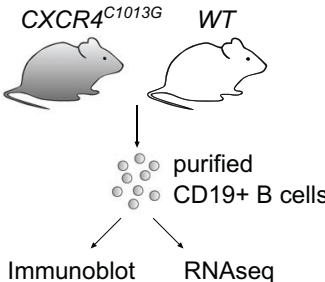

Immunoblot RNAseq b

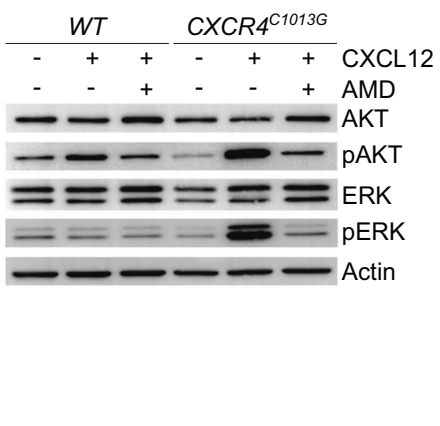

C

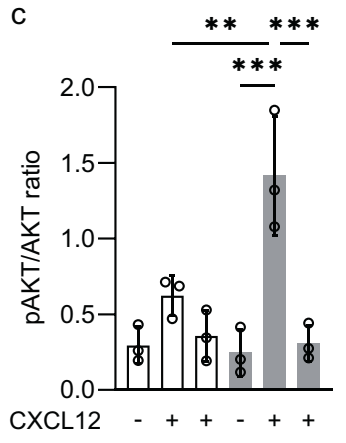

AMD3100 - - + - +

$\square W T$

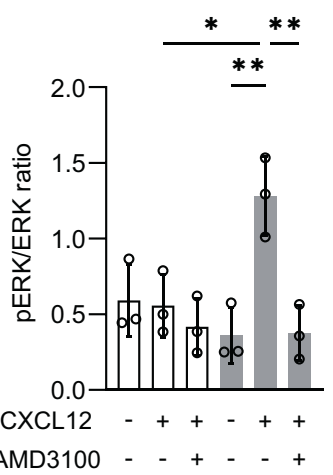

d

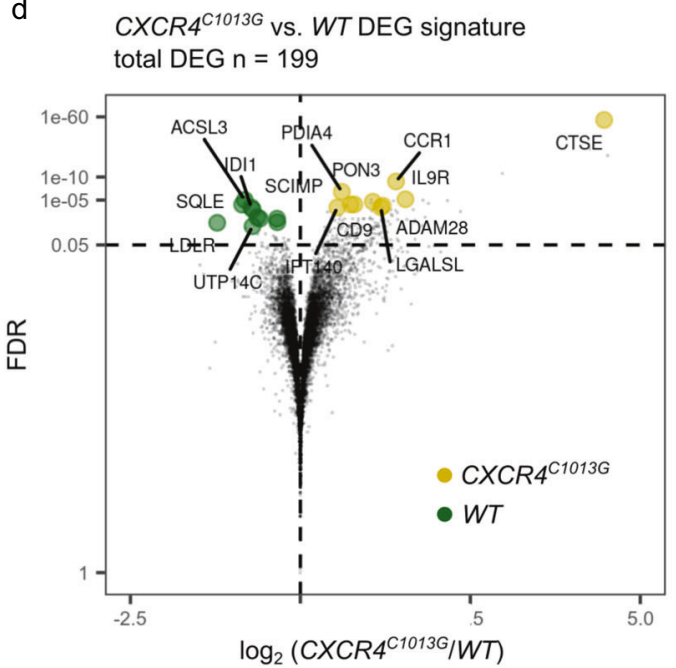

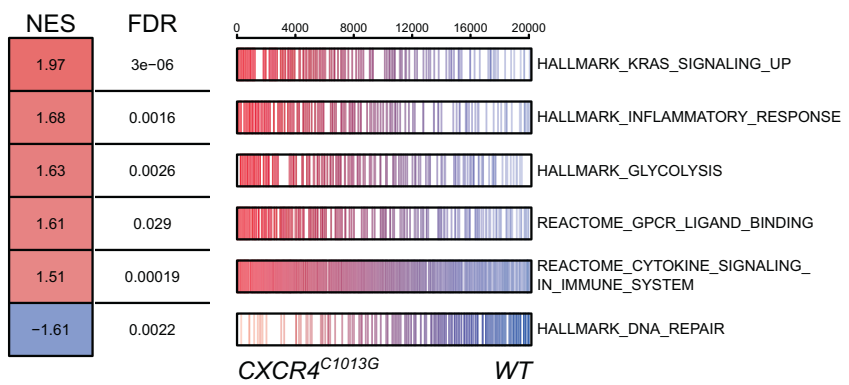

Fig. 1 Identification of a transcriptional signature of hyperactivated CXCR4 signaling. a Illustration of the $C X C R 4{ }^{C 1013 G}$ truncating mutation

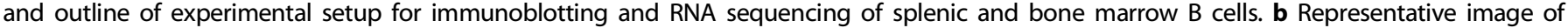
immunoblotting for AKT, pAKT (Ser473), ERK, pERK (Thr202/Tyr204), and Actin of CD19+ B cells treated with/without 50 nM CXCL12 and/or 10 $\mu$ M

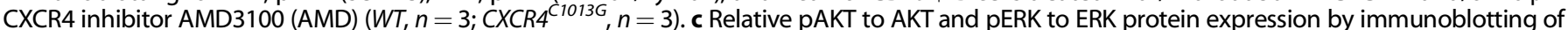
$W T$ and $C X C R 4^{C 1013 G}$ CD19+ B cells treated with/without $50 \mathrm{nM}$ CXCL12 and/or $10 \mu \mathrm{M}$ CXCR4 inhibitor AMD3100 (WT, $\left.\mathrm{n}=3 ; \mathrm{CXCR4}{ }^{\mathrm{C} 1013 G}, \mathrm{n}=3\right)$.

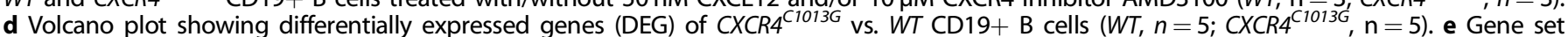
enrichment analysis (GSEA) of CXCR4 ${ }^{C 1013 G}$ vs. WT CD19+B cells showing normalized enrichment scores (NES) and false discovery rates (FDR) for

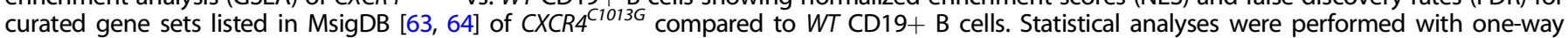
ANOVA with Tukey correction for multiple comparisons, ${ }^{*} P<0.05,{ }^{*} P<0.01,{ }^{* * *} P<0.001$. Error bars indicate standard deviation (SD).

readily increased upon stimulation with CXCL12 compared to control $B$ cells (Fig. 1b, c). To get a more comprehensive understanding to which extent CXCR4 hyperactivation shapes the transcriptional landscape, we performed whole-transcriptome profiling of CD19+ B cells from CXCR4 ${ }^{C 1013 G}$ and WT mice. CXCR4 ${ }^{C 1013 G} \mathrm{~B}$ cells exhibited a set of 199 differentially expressed genes (DEGs, protein coding, $p_{\text {adj }}$ $<0.05$; $\log \mathrm{FC}>0.5$ or $<-0.5$ ) compared to $W T$ controls (Fig. 1d). Among upregulated genes in B cells with hyperactivated CXCR4 signaling, we identified genes involved in chemokine signaling, migration and adhesion ( $C \mathrm{Cr} 1, C_{x C l 1} \mathrm{CxCl}_{2}$, Igfn1, Cntn2, Jaml), NOTCH signaling (Sorbs2), inflammation, and cytokine signaling (II9r, II7r, Csf2rb, Trem1), B-cell maturation (Rag1, Rag2), plasma cell differentiation and proliferation (Prdm1), metabolism $(P d k 1)$ and cell cycle progression (Nek6). Of note, the CXCL12-binding receptor Ackr3 (encoding CXCR7), which is known to form heterodimers with CXCR4 and is dysregulated in inflammatory diseases and cancers [32], was upregulated. From these significantly regulated $199 \mathrm{DEG}$ in CXCR4 ${ }^{C 1013 G}$ vs. WT, we generated a gene set defining the transcriptional profile of hyperactivated CXCR4 signaling (CXCR4a) in B cells (Supplementary Table T2). Subsequently, to test if CXCR4a represents a meaningful biological readout for enhanced CXCR4 signaling, we performed GSEA analysis with well-established published transcriptional signatures. We found that B cells of CXCR4 ${ }^{C 1013 G}$ mice enriched chemokine receptor signaling, inflammatory response and cytokine signaling pathways (Fig. 1e). Fully in line with the implication of CXCR4 in B-cell cancers, we found cancerrelevant pathways such as Kras signaling and glycolysis as well as depletion of DNA repair pathways in CXCR4 ${ }^{C 1013 G}$ B cells (Fig. 1e).

Thus, we here defined the transcriptional signature of hyperactivated CXCR4 signaling in B cells. Our data demonstrate the complexity of the transcriptional consequences of CXCR4 activation and represent a tool to assess activity of the CXCR4 pathway. Moreover, we found that enhancing CXCR4 signaling activity leads to the enrichment of several cancer-associated pathways, revealing that hyperactivated CXCR4 signaling might thereby predispose $\mathrm{B}$ cells for malignant transformation.

\section{Enhanced CXCR4 signaling cooperatively accelerates lymphoproliferation and promotes disease aggressiveness with TCL1}

CXCR4 plays an essential role in B-cell biology and is highly expressed in B cells as compared to all other cell types (Supplementary Fig. S2a). Considering that CXCR4 hyperactivation induced several cancer-relevant pathways in $B$ cells and 
confirming the association of elevated CXCR4 expression with adverse prognosis in CLL patients (Supplementary Fig. S2b), we sought to directly investigate the effect of enhanced CXCR4 signaling on B-cell lymphoproliferation and CLL development in vivo. We thus chose the $E \mu-T C L 1$ model of B-cell tumorigenesis, where the T-cell lymphoma/leukemia 1 (TCL1) transgene is targeted to B cells [21] and intercrossed it with CXCR4 ${ }^{C 1013 G}$ mice (Fig. 2a). As expected from previous experiments [21], $E \mu-T C L 1$ mice developed a gradually progressing B-cell lymphoproliferation with a CD19+CD5+/CD19+B220dim immunophenotype (Fig. 2b, Supplementary Fig. S3a). We found that in $E \mu-T C L 1 ; C X C R 4^{C 1013 G}$ mice, this lymphoproliferation started earlier as compared to $E \mu-T C L 1$, indicated by an increased bone marrow and splenic infiltration of CD19+CD5+/CD19+B220dim cells (Fig. 2b, Supplementary Fig. S3a) and increased spleen weight starting at 5-6 months of age (Fig. 2c). This finding was supported by histopathological analysis, where $E \mu-T C l 1$ mice exhibited enlarged spleens with moderate preservation of the white pulp and clear expansion of the red pulp, which was incipiently infiltrated by $\mathrm{B}$ cells, while in $E \mu$-TCL1;CXCR4 ${ }^{\text {C1013G }}$ mice the white pulp was atrophic with expansion of the red pulp and morphologically more pronounced infiltration (Fig. 2d, Supplementary Fig. S3b). In the bone marrow, the infiltration was significantly increased in the $E \mu-T C L 1 ; C X C R 4^{1013 G}$ compared to $E \mu$ TCL1 (Fig. 2d, Supplementary Fig. S3b). B220 expression at this stage was significantly lower in $E \mu-T C L 1 ; C X C R 4^{C 1013 G}$ compared to $E \mu-T C L 1$ CD19+CD5+ cells (Supplementary Fig. S3C). Importantly, the frequency of $\mathrm{CD} 3 \mathrm{e}+, \mathrm{CD} 4+$, and $\mathrm{CD} 8+\mathrm{T}$ cells in spleens of $E \mu$ $T C L 1$ and $E \mu-T C L 1 ; C X C R 4^{C 1013 G}$ did not differ significantly (Supplementary Fig. S3d). In summary, TCL1 mediated B-cell lymphoproliferation is accelerated by enhanced CXCR4 signaling.

To investigate if CXCR4 hyperactivation alone creates a predisposition favoring TCL1-driven B-cell proliferation and CLL development, we performed B-cell immunophenotyping of CXCR4 $4^{C 1013 G}$ mice, focusing on transitional $B$ cells and the population of CD19+/ B220dim/CD5 + B1 B cells. A potential mechanism of CLL development in the $E \mu-T C L 1$ mouse involves accumulation of autoreactive $B$ cells in the transitional T1 population, giving rise to CD19+/B220dim and, to limit autoreactivity, CD5 + B cells, which eventually progress to lethal B-cell leukemia [33-35]. Indeed, in spleens of CXCR4 ${ }^{\text {11013G }}$ mice, T1 B cells were increased, and T2 B cells were decreased (Fig. 2e). The CD19+B220dim/CD5+ B1 B-cell population known from E $\mu-T C L 1$ mice was already significantly increased in bone marrow and spleen of CXCR4 ${ }^{C 1013 G}$ mice compared to WT littermate controls (Fig. $2 \mathrm{f}$, Supplementary Fig. S3e, f), which was in line with the observed

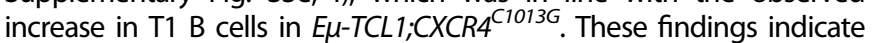
an inherent susceptibility of B cells with CXCR4 hyperactivation for aberrant B-cell lymphoproliferation, which is further enhanced in cooperation with TCL1.

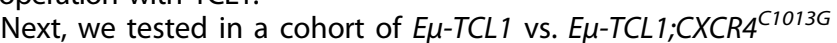
mice how CXCR4 hyperactivation impacts disease onset. We found that CXCR4 activation profoundly accelerates development of symptomatic CLL requiring euthanasia in collaboration with TCL1. Enhancing CXCR4 activity reduced the median survival by $\sim 100$ days compared to TCL1 alone (Fig. 3a). Importantly, this finding was accompanied by enhanced nodal dissemination of $\mathrm{CLL}$, as indicated by the development of pronounced lymphadenopathy, which was not observed in E $\mu$-TCL1 mice (Fig. 3b, Supplementary Fig. S4a). The immunophenotype and infiltration with $T$ cells remained unchanged in mice with symptomatic CLL requiring euthanasia, as seen in pre-malignant mice, and spleen and liver weights were similar when mice presented with symptoms (Supplementary Figs S4b, c). Histopathology of mice presenting with symptomatic CLL showed extensive infiltration of the red pulp in both genotypes, with complete atrophy of the white pulp in spleens. Visually evaluated by an experienced hematopathologist, the bone marrow of $E \mu-T C L 1 ; C X C R 4^{C 1013 G}$ mice displayed a diffuse infiltration pattern, compared to a more focal infiltration pattern in E $\mu$-TCL1 controls (Fig. 3c, Supplementary Fig. S4d). Strikingly, three out of seven $E \mu-T C L 1 ; C X C R 4^{C 1013 G}$ mice exhibited hallmark features of aggressive B-cell lymphoma in affected lymph nodes, morphologically resembling DLBCL-type Richter's transformation occurring in CLL patients. Accordingly, lymphoma cells were larger than typical $E \mu-T C L \quad C L L$ cells, infiltrated surrounding muscle and adipose tissue, and showed higher expression of the proliferation marker Ki67 (Fig. 3d and Supplementary Fig. S4e). Furthermore, three E $\mu$-TCL1;CXCR4 ${ }^{C 1013 G}$ mice presented with histiocytic sarcoma, a rare complication also in CLL patients, in which CLL cells transform into malignant histiocytes [36] (Supplementary Fig. S4f).

To investigate if the effects of CXCR4 hyperactivation on B-cell leukemia and lymphoma development and dissemination are $B$ cell intrinsic, we isolated splenocytes of pre-malignant animals and assessed their migratory capacity in response to CXCL12 ex vivo. When assessing the subgroup of CD19+CD5+ cells, of which $E \mu-T C L 1$ tumors evolve, we could see significantly higher migratory capacity specifically in E $\mu-T C L 1 ; C X C R 4^{C 1013 G}$ compared to $W T, E \mu-T C L 1$ and even $C X C R 4^{C 1013 G}$. Strikingly, no increased migratory potential compared to $C X C R 4^{C 1013 G}$ could be seen in bulk splenocytes and CD5+ T cells, suggesting a direct B-cellintrinsic collaboration of TCL1 and CXCR4 hyperactivation (Fig. 3e).

Thus, we provide first experimental in vivo evidence that CXCR4 hyperactivation supports and accelerates lymphoproliferation and CLL development in a susceptible genetic background, favoring development of a highly proliferative, nodally disseminating cancer with features of aggressive B-cell lymphoma or histiocytic sarcoma in a subset of $E \mu-T C L 1 ; C X C R 4^{C 1013 G}$ mice.

\section{Hyperactivated CXCR4 is a hallmark of aggressive lymphoma biology}

Next, we sought to determine if enhanced CXCR4 activity would also result in a more accelerated lymphoma biology in a model of aggressive lymphoma. Analyzing published datasets of $\mathrm{DLBCL}$, the most common aggressive B-cell lymphoma [27, 28, 37], we confirmed that elevated CXCR4 expression correlates with adverse prognosis (Supplementary Fig. S5a) and that CXCR4 mutations are present, but rare, in DLBCL patients (Supplementary Figure S5b and Supplementary Table T3). To functionally investigate the role of CXCR4 hyperactivation in the context of aggressive B-cell lymphoma, we chose the $E \mu-M y c$ mouse model, which is characterized by B-cell-targeted overexpression of MYC, leading to MYC-driven aggressive B-cell lymphoma [31]. This model reflects some of the features of MYC-dependent B-cell biology, which is activated in DLBCL and Burkitt's lymphoma. To investigate how additional CXCR4 activity alters MYC-induced lymphomagenesis, we intercrossed CXCR4 ${ }^{C 1013 G}$ with $E \mu-M y c$ mice (Fig. 4a). In young 1-month-old mice, we found similarly elevated

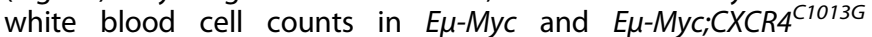
indicating tumor development in both cohorts (Supplementary Fig. S6a). Strikingly, even at this very early time point, we could already discover increased spleen weights compared to agematched EM-Myc and control animals (Fig. 4b). This finding was complimented by histopathology, showing that the spleens of $E \mu$ $M y c ; C X C R 4^{C 1013}$ mice had a more pronounced infiltration of premalignant B cells (Fig. $4 \mathrm{c}$ and Supplementary Fig. S6b) very early on. In mice with manifest lymphoma, the EM-Myc;CXCR4 $4^{C 1013 G}$ genotype was associated with a more aggressive presentation as demonstrated by larger spleens and higher bone marrow cell count (Fig. 4d, e). Although EM-Myc;CXCR4 ${ }^{C 1013 G}$ displayed features indicative of a more aggressive lymphoma phenotype, median survival did not differ compared to $E \mu-M y c$ controls (Fig. 4f). In mice with lymphoma requiring euthanasia, leukocyte counts and immunophenotype of tumors were comparable between both cohorts (Supplementary Fig. S6c, d). Histopathology confirmed the aforementioned findings of the pre-malignant cohorts, and revealed that $E \mu-M y c ; C X C R 4^{C 1013 G}$ mice presented with a higher 
a

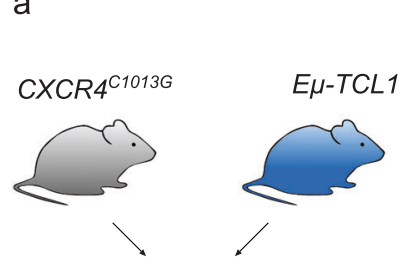

E $\mu$-TCL1;CXCR4 $4^{C 1013 G}$

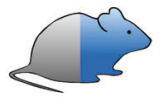

b

$E \mu-T C L 1$

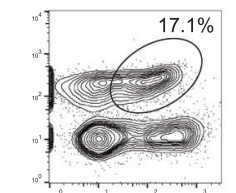

E $\mu-T C L 1 ; C X C R 4^{C 1013 G}$

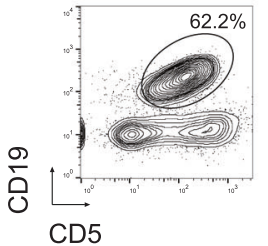

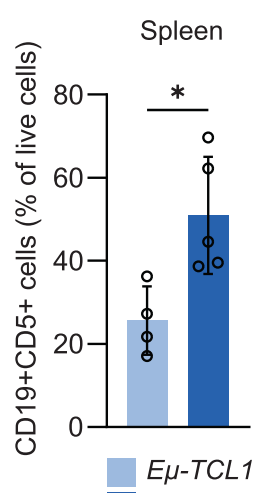

$E \mu-T C L 1 ; C X C R 4^{C 1013 G}$
C



$E \mu-T C L 1$

$E \mu-T C L 1 ; C X C R 4^{C 1013 G}$ d



e

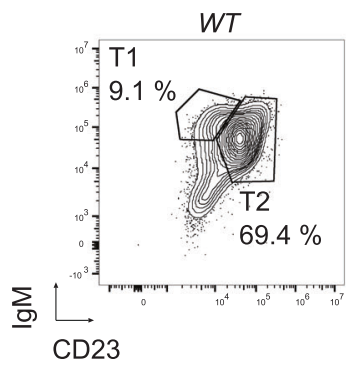

T1 $\mathrm{B}$ cells

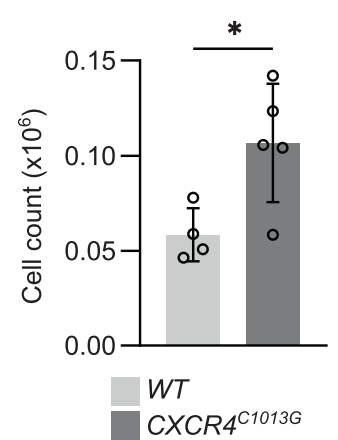

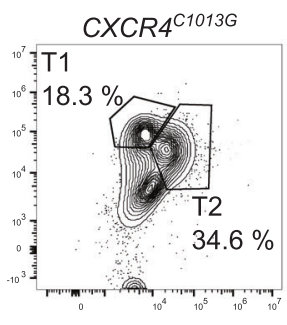

T2 B cells



f

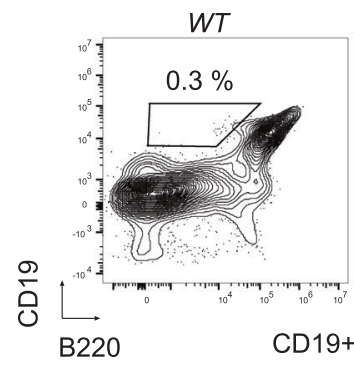

Bone Marrow



Bone Marrow
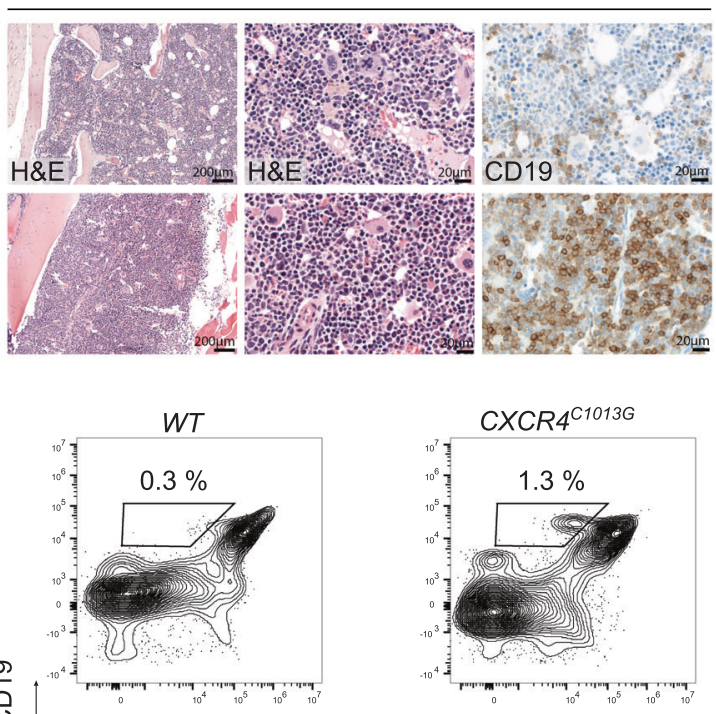

D19+B220dimCD5+



Fig. 2 CXCR4 hyperactivation blocks B-cell differentiation leading to expansion of pre-malignant B cells in cooperation with TCL1.

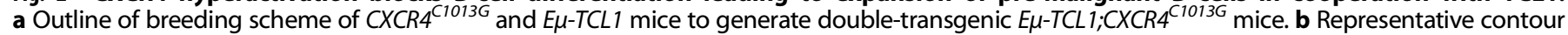
plots of splenic CD19+CD5+ cells by flow cytometry in 5-6-month-old animals and quantification of splenic and bone marrow CD19+CD5+ cells $\left(E \mu-T C L 1, n=4 ; E \mu-T C L 1 ; C X C R 4^{C 1013 G}, n=5\right)$. c Spleen weight of 5-6-month-old mice (E $\mu$-TCL1, $\left.n=4 ; E \mu-T C L 1 ; C X C R 4^{C 1013 G}, n=6\right)$. d Representative images of H\&E and immunohistochemistry of 5-6-month-old $E \mu-T C L 1$ and $E \mu-T C L 1 ; C X C R 4^{C 1013 G}$ mice (scale bars spleen: overview = 1 mm, detailed images: $200 \mu \mathrm{m}$, scale bars bone marrow: overview $=200 \mu \mathrm{m}$, detailed images $=20 \mu \mathrm{m})$. e Quantification and representative contour plots of splenic T1 (lgM+CD23-) and T2 (lgM+CD23+) cells gated on B220+CD93+ B cells by flow cytometry of 3-month-old animals $(W T$, $n=5$; CXCR4 $\left.{ }^{C 1013 G}, n=5\right)$. f Quantification and representative contour plots of splenic and bone marrow CD19+B220dimCD5+ B1 cells of 3-month-old animals $\left(W T, n=5 ; C X C R 4^{C 1013 G}, n=5\right)$. Statistical analyses were performed with Student's $t$ test, ${ }^{*} P<0.05,{ }^{* *} P<0.01,{ }^{* * *} P<0.001$. Error bars indicate standard deviation (SD). 


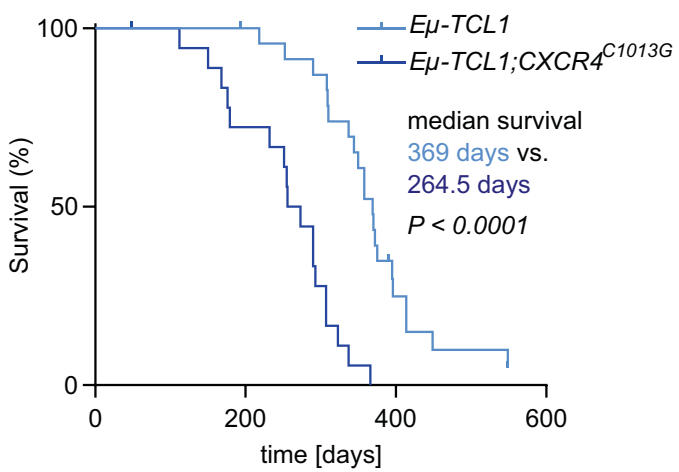

b

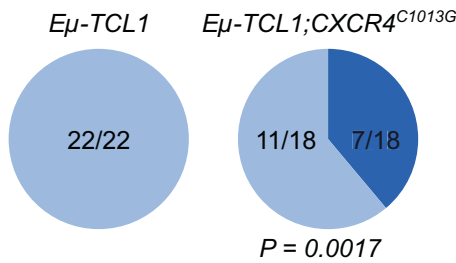

no lymphadenopathy

lymphadenopathy
C

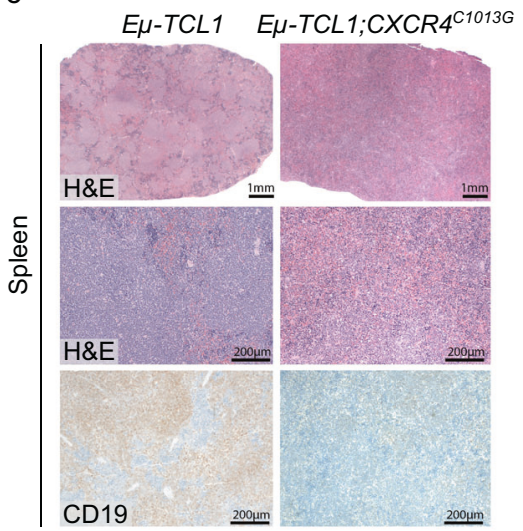

$E \mu-T C L 1 \quad E \mu-T C L 1 ; C X C R 4^{C 1013 G}$

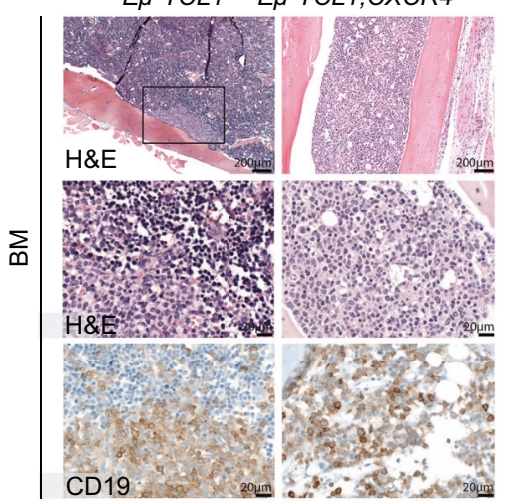

d

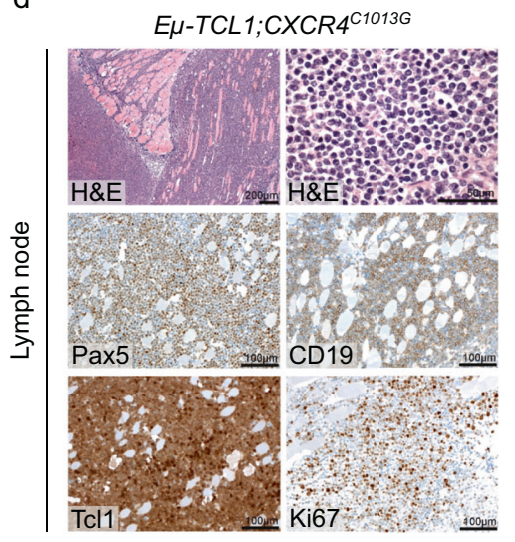

e

All splenocytes

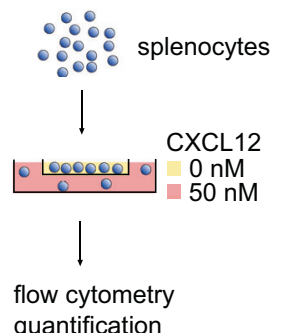

quantification
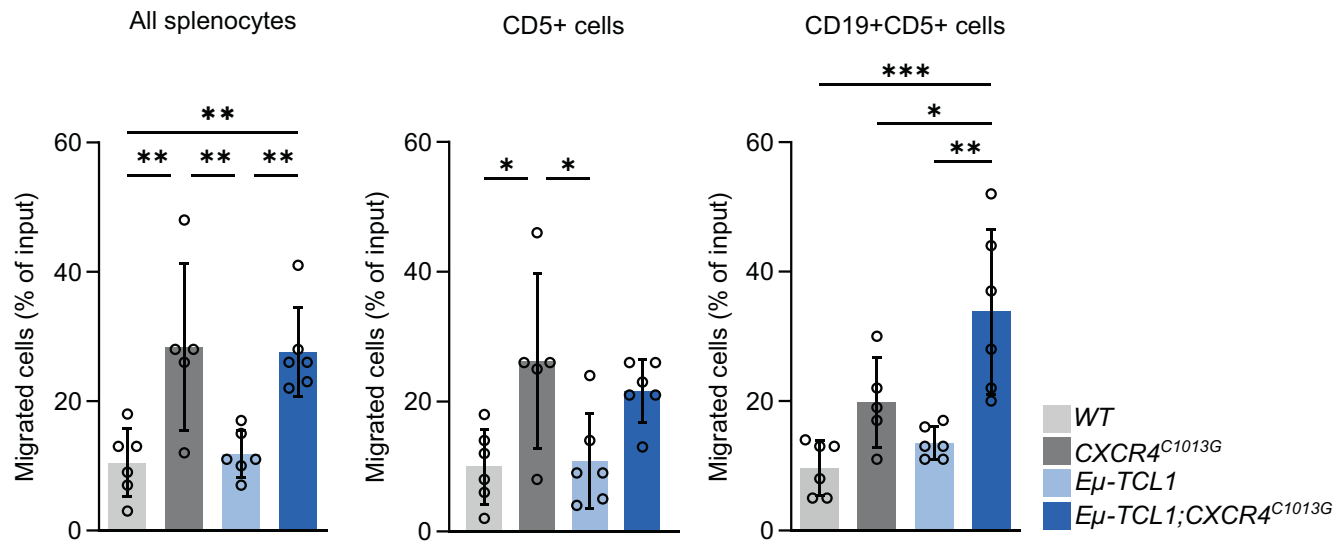

Fig. 3 CXCR4 hyperactivation accelerates TCL1-induced lymphomagenesis and dissemination in vivo. a Kaplan-Meier survival curves of the indicated cohorts of mice ( $E \mu-T C L 1, n=18 ; E \mu-T C L 1 ; C X C R 4^{C 1013 G}, n=22$ ). Median survival and $P$ value of logrank (Mantel-Cox) test is shown. b Pie

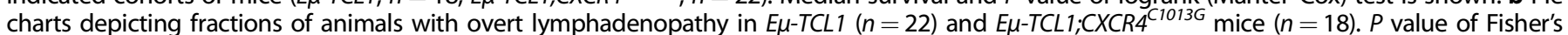

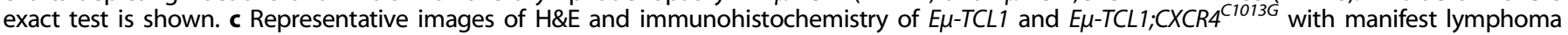
(scale bars spleen: overview $=1 \mathrm{~mm}$, detailed images: $200 \mu \mathrm{m}$, scale bars bone marrow: overview $=200 \mu \mathrm{m}$, detailed images $=20 \mu \mathrm{m}$ ). d Representative images of H\&E and immunohistochemistry of a E $\mu-T C L 1 ; C X C R 4^{C 1013 G}$ animal presenting with Ki67 positive Richter-like aggressive lymphoma (scale bars: H\&E overview $=200 \mu \mathrm{m}, \mathrm{H} \& \mathrm{E}$ detailed image $=50 \mu \mathrm{m}, \mathrm{IHC}$ detailed images $=100 \mu \mathrm{m}$ ). e Experimental setup and quantification for ex vivo transwell migration of all splenocytes, CD5 + T cells and CD19+CD5+ cells toward $50 \mathrm{nM}$ CXCL12 isolated from genotypes as indicated (WT, $\left.n=6 ; C X C R 4^{C 1013 G}, n=5 ; E \mu-T C L 1, n=6 ; E \mu-T C L 1 ; C X C R 4^{C 1013 G}, n=6\right)$. Statistical analyses were performed with one-way ANOVA with Tukey correction for multiple comparisons, ${ }^{*} P<0.05$, ${ }^{* *} P<0.01,{ }^{* *} P<0.001$. Error bars indicate standard deviation (SD).

tendency toward extranodal disease, e.g., hepatic infiltration by lymphoma cells, further indicating a more invasive, extranodal phenotype of CXCR4 hyperactivated lymphoma (Fig. $4 \mathrm{~g}$ and Supplementary Fig. S6e).

To investigate CXCR4 expression in E $\mu$-Myc mice as compared to $W T$ and $E \mu$-TCL1 mice as a possible reason for no additional effects of CXCR4 hyperactivation on survival in the E $\mu$-Myc lymphoma model, we first measured CXCR4 surface expression in the different genotypes. E $\mu$-Myc lymphoma cells displayed significantly increased CXCR4 surface expression compared to normal B cells and E $\mu$-TCL1 lymphoma cells (Supplementary Fig. S7a), indicating a potential link between MYC and CXCR4 expression. To further explore the association of MYC and CXCR4 expression, we analyzed patient datasets and found a significant correlation of MYC mRNA and IG-MYC translocation status with CXCR4 mRNA expression in B-cell lymphoma patients (Supplementary Fig. S7b, c). Furthermore, we performed RNA 
a

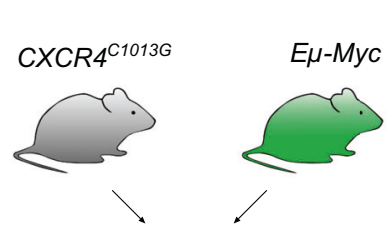

E $\mu-M y c ; C X C R 4^{C 1013 G}$

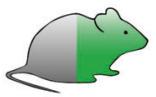

b

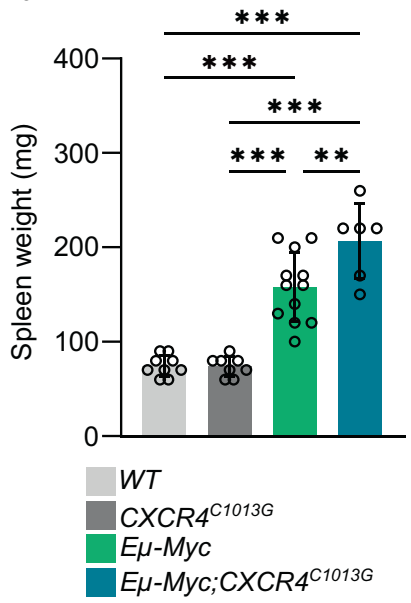

e

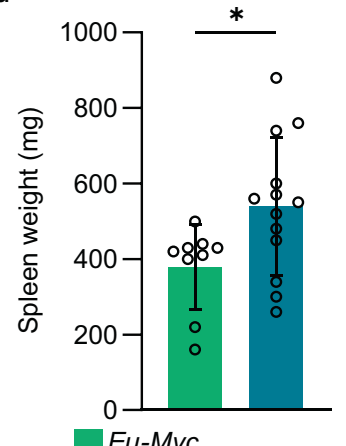

$E \mu-M y c$

$E \mu-M y c ; C X C R 4^{C 1013 G}$

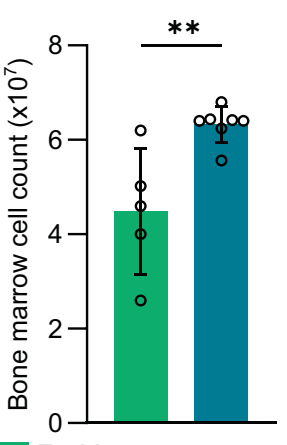

$E \mu-M y c$

E $\mu-M y c ; C X C R 4^{C 1013 G}$ g

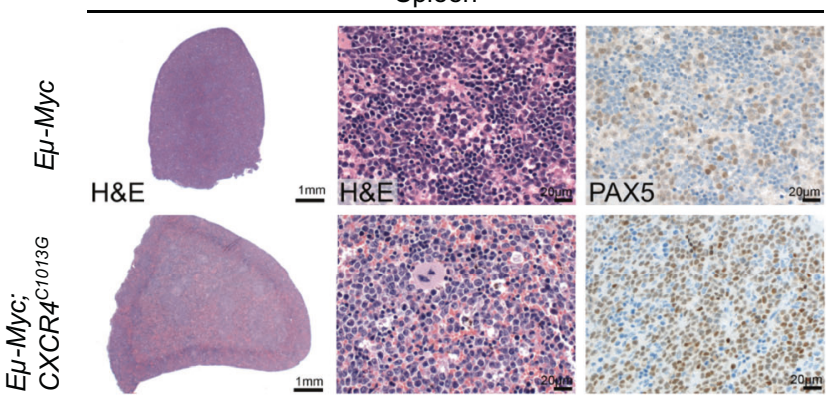



f

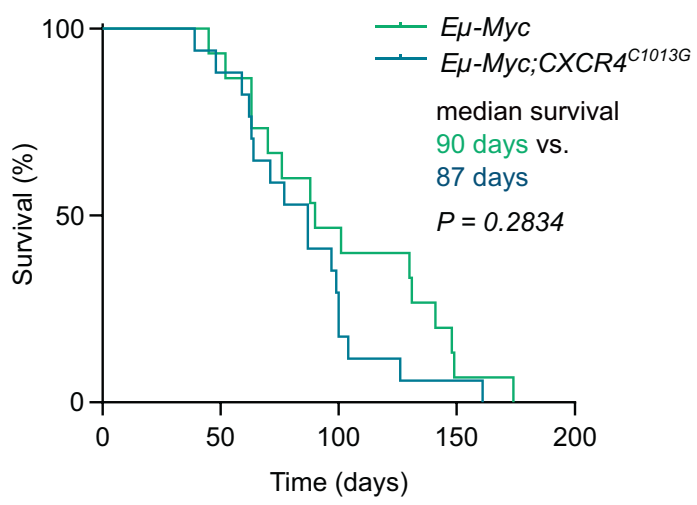

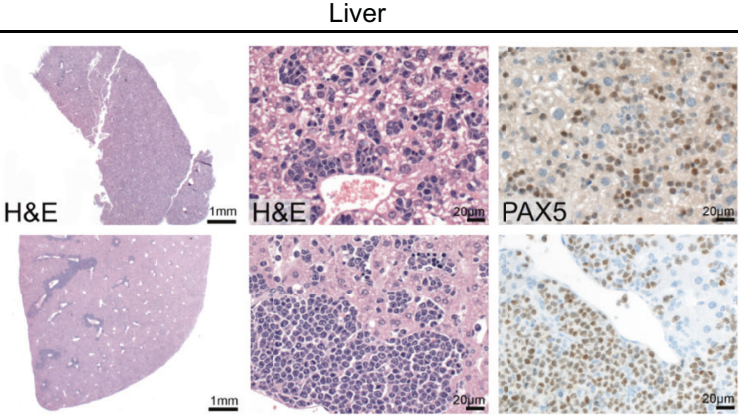

Fig. 4 CXCR4 hyperactivation does not accelerate MYC-driven B-cell lymphoma. a Outline of breeding scheme of $C X C R 4^{C 1013 G}$ and $E \mu-M y C$ mice to generate double-transgenic $E \mu-M y c ; C X C R 4^{C 1013 G}$ mice. $\mathbf{b}$ Spleen weight of 1-month-old animals of genotypes as indicated $(W T, n=9$; $\left.C X C R 4^{C 1013 G}, n=8 ; E \mu-M y c n=12 ; E \mu-M y c ; C X C R 4^{(1013 G}, n=6\right)$. c Representative images of H\&E and immunohistochemistry of 1-month-old E $\mu$ $M y c$ and $E \mu-M y c ; C X C R 4^{C 1013 G}$ mice (scale bars: overview $=1 \mathrm{~mm}$, detailed images: $\left.20 \mu \mathrm{m}\right)$. d Spleen weight of $E \mu-M y c(n=9)$ and $E \mu-M y c ;$ $C X C R 4^{C 1013 G}(n=13)$ mice with manifest lymphoma. e Bone marrow cell count of $E \mu-M y c(n=5)$ and $E \mu-M y c ; C X C R 4^{C 1013 G}(n=7)$ mice with manifest lymphoma. f Kaplan-Meier survival curves of the indicated cohorts of mice $\left(E \mu-M y c, n=15 ; E \mu-M y c ; C X C R 4^{C 1013 G}, n=17\right)$. Median survival and $P$ value of logrank (Mantel-Cox) test are shown. g Representative images of H\&E and immunohistochemistry of E $\mu$-Myc and E $\mu$ Myc;CXCR4 ${ }^{C 1013 G}$ mice with manifest lymphoma (scale bars spleen: overview $=1 \mathrm{~mm}$, detailed images: $20 \mu \mathrm{m}$, scale bars liver: overview $=$ $1 \mathrm{~mm}$, detailed images $=20 \mu \mathrm{m}$ ). Statistical analyses were performed with Student's $t$ test or one-way ANOVA with Tukey correction for multiple comparisons, ${ }^{*} P<0.05,{ }^{* *} P<0.01,{ }^{* *} P<0.001$. Error bars indicate standard deviation (SD).

sequencing on CD19+ lymphoma cells from $E \mu-M y c$ and $E \mu-M y c$; CXCR4 ${ }^{C 1013 G}$ and found that both groups displayed a highly similar transcriptome dominated by the MYC signature, with only eight pathways significantly altered in a gene set enrichment analysis (Supplementary Fig. S8a, b, c). In line with MYC-induced CXCR4 expression, applying our defined signature of CXCR4 hyperactivation did not show significant enrichment in the $E \mu-M y c ; C X C R 4^{C 1013 G}$ versus

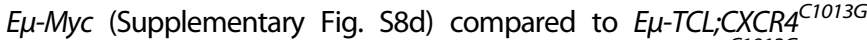
versus E $\mu$-TCL1 (Supplementary Fig. S8e). In E $\mu$-TCL;CXCR4 ${ }^{C 1013 G}$ mice the CXCR4 hyperactivation signature was highly and significantly enriched compared to $E \mu-T C L 1$ littermates. These findings indicate that MYC might positively regulate CXCR4 transcript levels in B-cell lymphoma, which could dampen the additional effect of CXCR4 hyperactivation by CXCR4 ${ }^{\text {C1013G }}$. 
We conclude that CXCR4 activation is an integrative hallmark of aggressive MYC-driven lymphoma, and show that MYC translocation and MYC expression correlate with increased CXCR4 expression. Accordingly, CXCR4 ${ }^{C 1013 G}$ in the E$\mu-M y c$ model did not further potentiate the already high transcriptional activity mediated by a MYC-CXCR4 axis and had no significant effect on lymphoma latency.

\section{Co-activation of CXCR4 and TCL1 governs a distinct oncogenic transcriptional program in B cells}

To further investigate how enhanced CXCR4 activation promotes B-cell lymphoproliferation in vivo, we isolated CD19+ B cells from

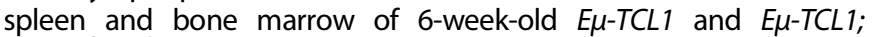
CXCR4 ${ }^{\mathrm{C1013G}}$ mice and respective controls and performed wholetranscriptome profiling (Fig. 5a). As expected, neither E $\mu$-TCL1 nor $E \mu-T C L 1 ; C X C R 4^{C 1013 G}$ had monoclonal B-cell proliferation at this stage detectable by transcriptomic clonality analysis (Supplementary Fig. S9) [38-40]. Gene set enrichment analysis of curated pathways

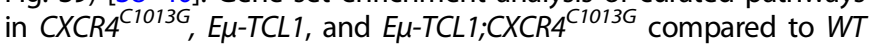
controls revealed an overlap of deregulated pathways (Fig. 5b). Importantly, pathways associated with B-cell cancer biology and inflammation were enriched and DNA repair pathways were depleted in E $\mu$-TCL1 compared to WT B cells, confirming the pre-

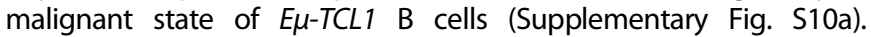
A subset of deregulated pathways were enriched specifically in $E \mu-T C L 1 ; C X C R 4^{C 1013 G}$ B cells (Fig. 5b). Among those, pathways involved in cell cycle progression (PLK1 pathway, G2M checkpoint, cell cycle checkpoints) were enriched, whereas pathways including p53 signaling and apoptosis (P53 pathway, P53 dependent G1 DNA damage response, apoptosis) and immune response (interferon gamma response) were depleted in $E \mu-T C L 1 ; C X C R 4^{C 1013 G}$ B cells.

Next, to gain further insight into the phenotypic differences and disease acceleration caused by additional CXCR4 signaling, we

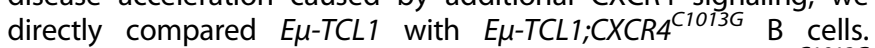
Strikingly, we found that the transcriptome of $E \mu-T C L 1 ; C X C R 4^{C 1013 G}$ $B$ cells maintained a distinct profile even compared to $E \mu-T C L 1$, comprising enrichment of cell cycle progression and proliferation pathways and depletion of p53-, apoptosis-, and immune response-related pathways (Fig. 5c). Analyzing expression of individual genes, we found 2140 differentially expressed genes between pre-malignant $\mathrm{B}$ cells from $E \mu-T C L 1$ vs. $E \mu-T C L 1$; $C X C R 4^{C 1013 G}$ mice. In line with the gene set enrichment analysis, cell cycle genes like cyclins (Ccne1), cyclin-dependent kinase phosphatases (Cdc25b), mitotic regulators (Aurkb, Prc1, Hdac6, Nek2, Mki67), DNA repair and DNA damage response genes (Brca2, Fanca, Rad54I) were among the top differentially expressed genes. Transcripts of well-established tumor suppressor genes (KIf6, CD82) and positive regulators of apoptosis (Pmaip1) were found to be suppressed in $E \mu-T C L 1 ; C X C R 4^{C 1013 G}$ (Fig. 5d). Remarkably, multiple key components of the Plk1-Foxm1 pathway were among the top upregulated genes, including Plk1, Foxm 1 itself, the bona-fide Foxm 1 target Cdc25b, the Foxm1 deubiquitinating enzyme Usp5 involved in stabilization of Foxm1 protein [41], and Myb, which forms a complex with Foxm 1 and is required for its function.

To further support the finding that CXCR4 hyperactivation potentiates oncogenic programs, we used the Cancer Gene Census [42] and human B-cell lymphoma datasets $[27,28]$ for cross-species

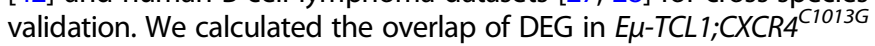
vs. EM-TCL1 B cells with the Cancer Gene Census (Supplementary Fig. $\mathrm{S} 10 \mathrm{~b})$ and $\mathrm{DLBCL}$ driving genes in two large patient cohorts (Supplementary Fig. S10c) and found that ten DEGs in our cohort were identified in both DLBCL datasets $[27,28]$.

To further evaluate if the transcriptional program of patients with Richter's transformation resembles the transcriptomic changes supported by enhanced CXCR4 signaling, we generated a gene signature from CLL patient data with Richter's transformation [43]. Strikingly, this "Richter-signature" enriched significantly only in $E \mu$ -

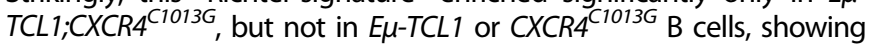
that key transcriptomic features of Richter's transformation are promoted by CXCR4 hyperactivation in cooperation with TCL1 (Fig. 5e and Supplementary Fig. S10d). Finally, we stratified a cohort of CLL patients using upregulated genes of the CXCR4 ${ }^{C 1013 G}$ vs. WT (CXCR4a) and $E \mu-T C L 1 ; C X C R 4^{C 1013 G}$ vs $E \mu-T C L 1$ signature. We found that patients characterized by CXCR4a had significantly reduced time to treatment

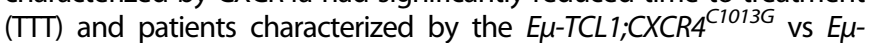
$T C L 1$ signature had significantly reduced $T T$ and overall survival (Fig. 5f), further supporting the association of CXCR4 hyperactivation with aggressive lymphoma biology.

\section{DISCUSSION}

In this study we co-expressed $C X C R 4^{C 1013 G}$ with the B-cell oncogenes $T C L 1$ and MYC to investigate the effects of enhanced CXCR4 signaling on B-cell leukemogenesis and lymphomagenesis. Hyperactivated CXCR4 functioned as an oncogene cooperating with TCL1 to accelerate CLL progression and development of aggressive B-cell lymphoma.

CXCR4 expression has been implicated as an adverse prognostic factor in DLBCL in retrospective analyses [14, 44]. However, CXCR4 signaling activity is a highly dynamic and tightly regulated process, which is not always adequately reflected by gene expression alone [5]. Previous studies on CXCR4 gain-of-function mutations focused on established Waldenström Macroglobulinemia cell lines, as CXCR4 mutations are present in a third of patients with this disease [45], thereby limiting conclusions to already transformed cells $[46,47]$. The role of CXCR4 in CLL has been thoroughly investigated and it has become clear that the CXCR4-CXCL12 axis is essential for CLL cells and their interaction with the microenvironment, especially in response to BTK inhibition with ibrutinib [17, 25]. However, it was unclear how hyperactive CXCR4 signaling impacts the development of CLL in vivo. Our work represents the first study providing in vivo evidence that CXCR4 acts as an oncogene in B cells by expressing a gain-of-function mutation together with known B-cell oncogenes. Importantly, CXCR4 ${ }^{\mathrm{C} 1013 \mathrm{G}}$ provokes tremendous transcriptomic and phenotypic changes in pre-malignant $B$ cells. We discovered an expansion of B1 B cells mediated by CXCR4 ${ }^{\text {C1013G, }}$ indicating a susceptibility of hyperactivated CXCR4 signaling toward mature B-cell neoplasms, as this fraction of B cells harbor the potential for transformation into CLL and mantle cell lymphoma [48]. This susceptibility does not result in development of B-cell neoplasms in the $C X C R 4^{C 1013 G}$ mouse model alone [9] but requires the context of additional oncogenic drivers, as shown in our studies. In cooperation with the oncogene TCL1, CXCR4 hyperactivation even favors development of aggressive B-cell lymphoma. Intriguingly, in CLL patients with Richter's transformation mutations in regulatory elements of CXCR4 have recently been discovered [43].

The transcriptional alterations induced by CXCR4 hyperactivation were consistent with CXCR4 function as indicated by enrichment of curated pathway gene sets for chemokine signaling and inflammation. Intriguingly, RNA sequencing also revealed enhanced expression of $\operatorname{Prdm} 1$, a master regulator of plasma cell proliferation and differentiation, which was found to be essential for the survival of WM cells by regulating EZH2, a promising therapeutic target in B-cell lymphoma $[49,50]$.

Of note, no mouse model with B-cell-specific CXCR4 ${ }^{C 1013 G}$ expression exists, therefore additional, non-B-cell-intrinsic effects of transgene expression in the microenvironment need to be taken into consideration. It cannot be completely ruled out that acceleration of lymphoma development might be partially mediated by activated CXCR4 signaling in T cells or other cell types of the tumor microenvironment known to express CXCR4. Importantly, all transcriptomic data shown originate from CD19purified $B$ cells. The finding that CXCR4 gene expression is highest in $B$ cells, and data that identified CXCR4 as a major regulator of B-cell development and function [2, 10, 51, 52] further support the notion of a B-cell-intrinsic effect of CXCR4 signaling in our models. Furthermore, the migratory capacity ex vivo, without any 
a

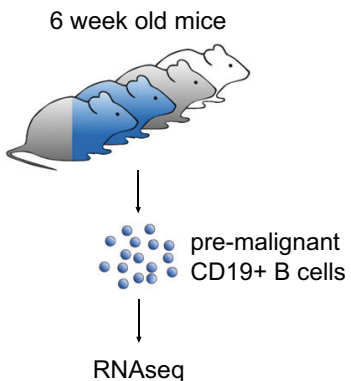

b

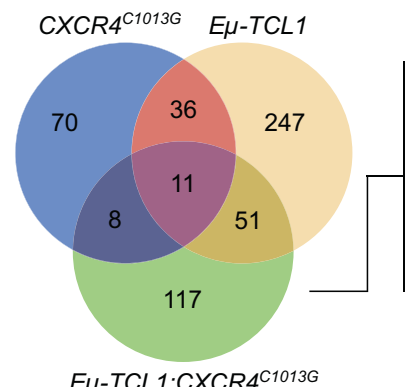

PID PLK1 PATHWAY

HALLMARK_G2M_CHECKPOINT

REACTOME_APOPTOSIS

REACTOME CELL CYCLE CHECKPOINTS

HALLMARK P53 PATHWAY

REACTOME_P53_DEPDENDANT_G1_DNA_

DAMAGE RESPONNSE

HALLMARK_INTERFERON_GAMMA_RESPONSE
C

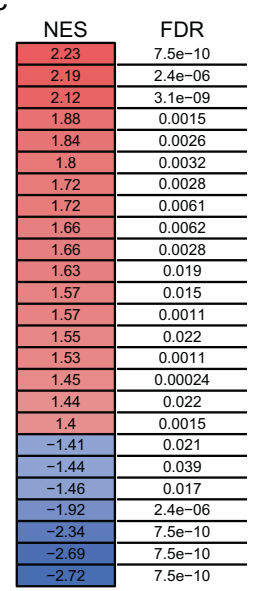

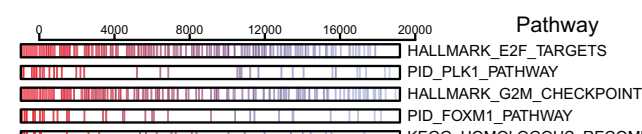

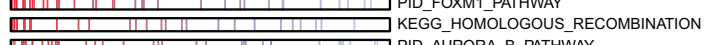

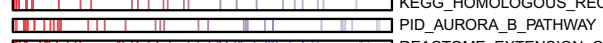

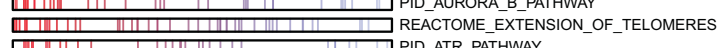
PID_ATR_PATHWAY KEGG_DNA_REPLICATION
REACTOME_CHROMOSOME_MAINTENANCE BIOCARTA_G2_PATHWAY REACTOME_DNA_STRAND_ELONGATION

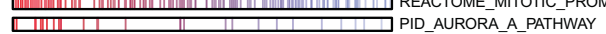
REACTOME_DNA_REPAIR REACTOME_CELL_CYCLE
REACTOME_TELOMERE_MAINTENANCE
REACTOME_CELL_CYCLE_MITOTIC PID_P53_DOWNSTREAM_PATHWAY PID_FOXO_PATHWAY

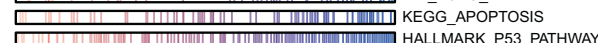
HALLMARK_P53_PATHWAY
HALLMARK_APOPTOSIS
HALLMARK_INTERFERON_GAMMA_RESPONSE $E \mu-T C L 1 ; C X C R 4^{C 1013 G} \quad E \mu-T C L 1$ d

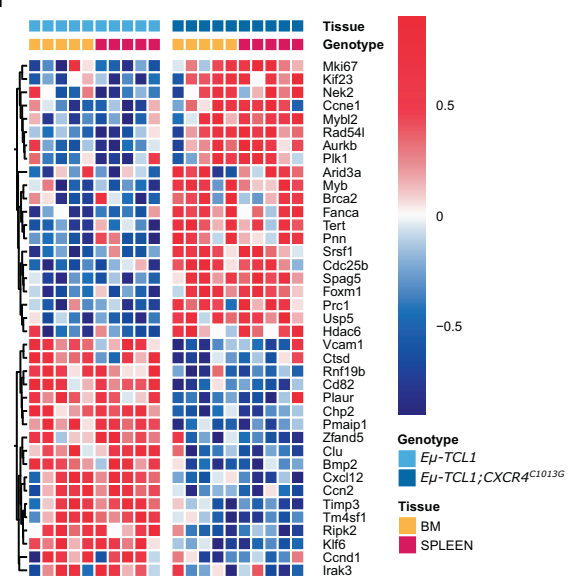

e

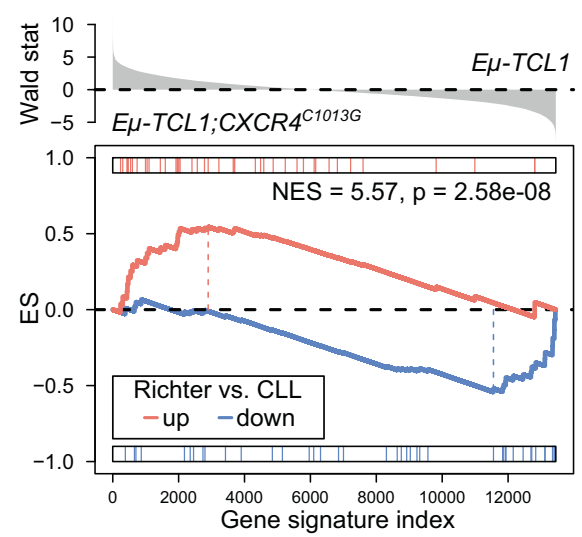

$f$

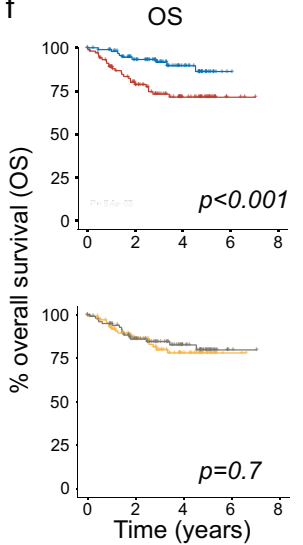

TTT

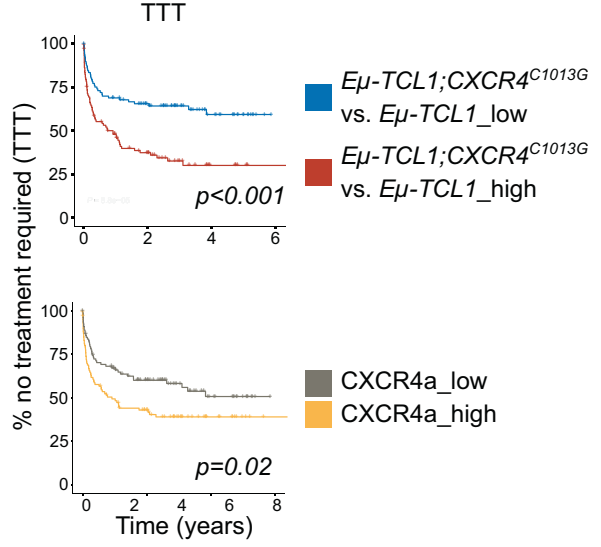

Fig. 5 Co-activation of CXCR4 and TCL1 governs a distinct oncogenic transcriptional program in B cells. a Outline of experimental setup for RNA sequencing of splenic and bone marrow pre-malignant $\mathrm{B}$ cells $\left(W T, n=5 ; C X C R 4^{C 1013 G}, n=5 ; E \mu-T C L 1, n=5 ; E \mu-T C L 1 ; C X C R 44^{C 1013 G}, \mathrm{n}=5\right)$. b Venn diagram showing overlap of significantly enriched pathways (padj <0.05) for curated gene sets listed in MsigDB [63, 64] of indicated

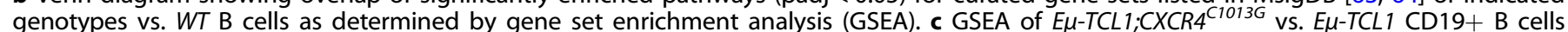
showing normalized enrichment scores (NES) and false discovery rates (FDR) for curated gene sets listed in MsigDB [63, 64]. d Selection of

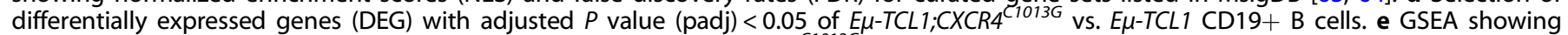
enrichment of a Richter's transformation signature [43] in E $\mu-T C L 1 ; C X C R 4^{C 1013 G}$ B cells. $f$ Kaplan-Meier plots of overall survival (OS) and time to treatment (TTT) in a cohort of CLL patients, for patients with high vs. low enrichment of upregulated genes from CXCR4 signatures (CXCR4 ${ }^{C 1013 G}$

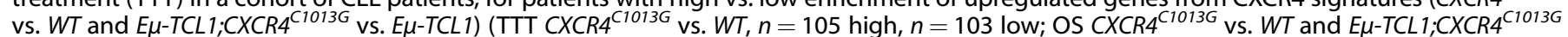
vs. $E \mu-T C L 1, \mathrm{n}=105$ in each group; TTT $E \mu-T C L 1 ; C X C R 4^{C 1013 G}$ vs. $E \mu-T C L 1, n=104$ in each group). $P$ values of logrank (Mantel-Cox) test are shown.

support of the microenvironment, is enhanced most prominently in CD19+CD5+ B cells of double-transgenic E $\mu$-TCL1;CXCR4 ${ }^{\text {C1013G }}$ animals compared to controls, suggesting a direct collaboration of TCL1 and CXCR4 in a specific subset of B cells. Of note, frequency of

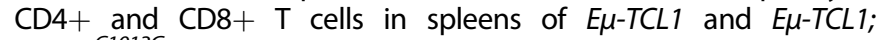
CXCR4 ${ }^{C 1013 G}$ did not differ significantly, which might suggest that CXCR4 activation in T cells did not result in dramatically reduced Tcell-mediated immune surveillance. Moreover, we could detect an aggressive lymphoma phenotype in the histology of a subset of mice as well as an enrichment of a patient-derived "Richtersignature" in the transcriptomic program of pre-malignant $E \mu-T C L 1$; CXCR4 ${ }^{\text {C1013G }} \mathrm{B}$ cells. This further indicates a B-cell-specific influence of CXCR4 signaling toward a more aggressive lymphoma phenotype. In the light of recent findings linking active Akt signaling, a downstream target of CXCR4, to Richter's transformation [23], it seems possible that Akt phosphorylation by hyperactivated CXCR4 is 
a contributing factor for the aggressive lymphoma phenotype in our CXCR4 hyperactivation models.

Using the E $\mu-M y c$ lymphoma model, we showed that CXCR4 transcript and surface expression is enhanced by oncogenic MYC. MYC can bind and activate the CXCR4 promoter [53], but this connection has not been explored in B-cell lymphoma so far.

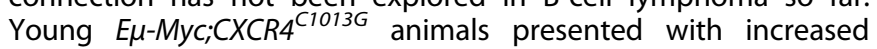
spleen weight and more pronounced lymphoproliferation, which did not translate in a reduction in overall survival. This could be explained by a proportion of transformed B cells depending fully on the MYC-oncogenic program and exponential tumor growth in $E \mu-M y c$ animals, which could mask biologically relevant effects of additional CXCR4 hyperactivation as used in our experimental approach. Furthermore, data from large B-cell lymphoma patient datasets revealed a correlation of MYC and CXCR4 expression and an increase of CXCR4 expression in patient samples with translocation of MYC further supporting a regulation of CXCR4 by MYC in a B-cell oncogenic context.

The cooperation of CXCR4 and TCL1 was associated with transcriptional activation of the Foxm1-Plk1 axis and proliferative pathways [54]. PI3K and Akt might be the central signaling node connecting CXCR4 and TCL1 signaling, resulting in inhibition of Foxo3 and de-repression of Foxm1 [55, 56]. In addition, PI3K/Akt phosphorylates Plk1 that engages in a feed-forward loop with Foxm1 [57]. Both Plk1 and Foxm1 are promising therapeutic targets in B-cell lymphoma $[58,59]$. The Myb oncogene, which also displays increased

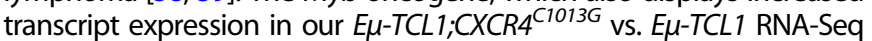
data, is crucial for Foxm1 function [60, 61], and the Myb-Foxm1 interaction is required for proliferation in the germinal centers, a site prone for malignant transformation of B cells [62].

In summary, we identified hyperactivated CXCR4 signaling as a cooperative oncogenic factor in B-cell leukemogenesis and lymphomagenesis associated with a distinct transcriptional program. Hyperactivated CXCR4 resulted in a more disseminated lymphoma phenotype and accelerated disease in TCL1-driven CLL, while also favoring development of aggressive lymphoma. Next to its role as a prognostic factor, CXCR4 activation might serve as potential biomarker for novel targeted therapies, e.g., therapies targeting the FOXM1-PLK1 axis and EZH2. These findings warrant further investigation.

\section{DATA AVAILABILITY}

The RNA-Seq data reported in this study are available on the National Center for Biotechnology Information's Gene Expression Omnibus under the accession code GSE178959.

\section{REFERENCES}

1. Nagasawa T, Hirota S, Tachibana K, Takakura N, Nishikawa S, Kitamura Y, et al. Defects of B-cell lymphopoiesis and bone-marrow myelopoiesis in mice lacking the CXC chemokine PBSF/SDF-1. Nature. 1996;382:635-8.

2. Freitas C, Wittner M, Nguyen J, Rondeau V, Biajoux V, Aknin ML, et al. Lymphoid differentiation of hematopoietic stem cells requires efficient Cxcr4 desensitization. J Exp Med. 2017;214:2023-40.

3. Ding L, Morrison SJ. Haematopoietic stem cells and early lymphoid progenitors occupy distinct bone marrow niches. Nature. 2013;495:231-5.

4. Scala S. Molecular pathways: targeting the CXCR4-CXCL12 axis-untapped potential in the tumor microenvironment. Clin Cancer Res. 2015;21:4278-85.

5. Busillo JM, Benovic JL. Regulation of CXCR4 signaling. Biochim Biophys Acta. 2007;1768:952-63.

6. Haribabu B, Richardson RM, Fisher I, Sozzani S, Peiper SC, Horuk R, et al. Regulation of human chemokine receptors CXCR4. Role of phosphorylation in desensitization and internalization. J Biol Chem. 1997;272:28726-31.

7. Hernandez PA, Gorlin RJ, Lukens JN, Taniuchi S, Bohinjec J, Francois F, et al. Mutations in the chemokine receptor gene CXCR4 are associated with WHIM syndrome, a combined immunodeficiency disease. Nat Genet. 2003;34:70-4.

8. Balabanian K, Lagane B, Pablos JL, Laurent L, Planchenault T, Verola O, et al. WHIM syndromes with different genetic anomalies are accounted for by impaired CXCR4 desensitization to CXCL12. Blood. 2005;105:2449-57.
9. Balabanian K, Brotin E, Biajoux V, Bouchet-Delbos L, Lainey E, Fenneteau O, et al. Proper desensitization of CXCR4 is required for lymphocyte development and peripheral compartmentalization in mice. Blood. 2012;119:5722-30.

10. Allen CD, Ansel KM, Low C, Lesley R, Tamamura H, Fujii N, et al. Germinal center dark and light zone organization is mediated by CXCR4 and CXCR5. Nat Immunol. 2004:5:943-52.

11. Wester HJ, Keller U, Schottelius M, Beer A, Philipp-Abbrederis K, Hoffmann F, et al. Disclosing the CXCR4 expression in lymphoproliferative diseases by targeted molecular imaging. Theranostics. 2015;5:618-30.

12. Mayerhoefer ME, Jaeger U, Staber $P$, Raderer M, Wadsak W, Pfaff $S$, et al. [68Ga] Ga-pentixafor PET/MRI for CXCR4 imaging of chronic lymphocytic leukemia: preliminary results. Investig Radio. 2018;53:403-8.

13. Herhaus P, Lipkova J, Lammer F, Yakushev I, Vag T, Slotta-Huspenina J, et al. CXCR4-targeted PET imaging of central nervous system B-cell lymphoma. J Nucl Med. 2020;61:1765-71.

14. Moreno MJ, Bosch R, Dieguez-Gonzalez R, Novelli S, Mozos A, Gallardo A, et al. CXCR4 expression enhances diffuse large $B$ cell lymphoma dissemination and decreases patient survival. J Pathol. 2015;235:445-55.

15. Ganghammer S, Gutjahr J, Hutterer E, Krenn PW, Pucher S, Zelle-Rieser C, et al. Combined CXCR3/CXCR4 measurements are of high prognostic value in chronic lymphocytic leukemia due to negative co-operativity of the receptors. Haematologica. 2016;101:e99-102.

16. Burger JA, Burger M, Kipps TJ. Chronic lymphocytic leukemia B cells express functional CXCR4 chemokine receptors that mediate spontaneous migration beneath bone marrow stromal cells. Blood. 1999;94:3658-67.

17. Burger JA, Tsukada N, Burger M, Zvaifler NJ, Dell'Aquila M, Kipps TJ. Blood-derived nurse-like cells protect chronic lymphocytic leukemia $B$ cells from spontaneous apoptosis through stromal cell-derived factor-1. Blood. 2000;96:2655-63.

18. Mohle R, Failenschmid C, Bautz F, Kanz L. Overexpression of the chemokine receptor CXCR4 in B cell chronic lymphocytic leukemia is associated with increased functional response to stromal cell-derived factor-1 (SDF-1). Leukemia. 1999;13:1954-9.

19. Recasens-Zorzo C, Cardesa-Salzmann T, Petazzi P, Ros-Blanco L, Esteve-Arenys A, Clot $\mathrm{G}$, et al. Pharmacological modulation of CXCR4 cooperates with BET bromodomain inhibition in diffuse large B-cell lymphoma. Haematologica. 2019;104:778-88.

20. Chen L, Ouyang J, Wienand K, Bojarczuk K, Hao Y, Chapuy B, et al. CXCR4 upregulation is an indicator of sensitivity to B-cell receptor/PI3K blockade and a potential resistance mechanism in B-cell receptor-dependent diffuse large B-cell lymphomas. Haematologica. 2020;105:1361-8.

21. Bichi R, Shinton SA, Martin ES, Koval A, Calin GA, Cesari R, et al. Human chronic lymphocytic leukemia modeled in mouse by targeted TCL1 expression. Proc Natl Acad Sci USA. 2002;99:6955-60.

22. Harris AW, Pinkert CA, Crawford M, Langdon WY, Brinster RL, Adams JM. The E mu-myc transgenic mouse. A model for high-incidence spontaneous lymphoma and leukemia of early B cells. J Exp Med. 1988;167:353-71.

23. Kohlhaas V, Blakemore SJ, Al-Maarri M, Nickel N, Pal M, Roth A, et al. Active Akt signaling triggers CLL toward Richter transformation via overactivation of Notch1. Blood. 2021;137:646-60.

24. Knittel G, Rehkamper T, Korovkina D, Liedgens P, Fritz C, Torgovnick A, et al. Two mouse models reveal an actionable PARP1 dependence in aggressive chronic lymphocytic leukemia. Nat Commun. 2017;8:153.

25. Chen SS, Chang BY, Chang S, Tong T, Ham S, Sherry B, et al. BTK inhibition results in impaired CXCR4 chemokine receptor surface expression, signaling and function in chronic lymphocytic leukemia. Leukemia. 2016;30:833-43.

26. Andritsos LA, Byrd JC, Cheverton P, Wu J, Sivina M, Kipps TJ, et al. A multicenter phase 1 study of plerixafor and rituximab in patients with chronic lymphocytic leukemia. Leuk Lymphoma. 2019;60:3461-9.

27. Reddy A, Zhang J, Davis NS, Moffitt AB, Love CL, Waldrop A, et al. Genetic and functional drivers of diffuse large $B$ cell lymphoma. Cell. 2017;171:481-94 e15.

28. Chapuy B, Stewart C, Dunford AJ, Kim J, Kamburov A, Redd RA, et al. Molecular subtypes of diffuse large $B$ cell lymphoma are associated with distinct pathogenic mechanisms and outcomes. Nat Med. 2018;24:679-90.

29. Crowther-Swanepoel D, Qureshi M, Dyer MJ, Matutes E, Dearden C, Catovsky D, et al. Genetic variation in CXCR4 and risk of chronic lymphocytic leukemia. Blood. 2009;114:4843-6.

30. Klintman J, Appleby N, Stamatopoulos B, Ridout K, Eyre TA, Robbe P, et al. Genomic and transcriptomic correlates of Richter transformation in chronic lymphocytic leukemia. Blood. 2021;137:2800-16.

31. Adams JM, Harris AW, Pinkert CA, Corcoran LM, Alexander WS, Cory S, et al. The c-myc oncogene driven by immunoglobulin enhancers induces lymphoid malignancy in transgenic mice. Nature. 1985;318:533-8.

32. Sanchez-Martin L, Sanchez-Mateos P, Cabanas C. CXCR7 impact on CXCL12 biology and disease. Trends Mol Med. 2013;19:12-22.

33. Hippen KL, Tze LE, Behrens TW. CD5 maintains tolerance in anergic B cells. J Exp Med. 2000;191:883-90. 
34. Nganga VK, Palmer VL, Naushad H, Kassmeier MD, Anderson DK, Perry GA, et al. Accelerated progression of chronic lymphocytic leukemia in Emu-TCL1 mice expressing catalytically inactive RAG1. Blood. 2013;121:3855-66. S1-16

35. Alankus B, Ecker V, Vahl N, Braun M, Weichert W, Macher-Goppinger S, et al. Pathological RANK signaling in B cells drives autoimmunity and chronic lymphocytic leukemia. J Exp Med. 2021;218:e20200517.

36. Shao H, Xi L, Raffeld M, Feldman AL, Ketterling RP, Knudson R, et al. Clonally related histiocytic/dendritic cell sarcoma and chronic lymphocytic leukemia/small lymphocytic lymphoma: a study of seven cases. Mod Pathol. 2011;24:1421-32.

37. Morin RD, Mungall K, Pleasance E, Mungall AJ, Goya R, Huff RD, et al. Mutational and structural analysis of diffuse large B-cell lymphoma using whole-genome sequencing. Blood. 2013;122:1256-65.

38. Shugay M, Bagaev DV, Turchaninova MA, Bolotin DA, Britanova OV, Putintseva EV, et al. VDJtools: unifying post-analysis of T cell receptor repertoires. PLoS Comput Biol. 2015;11:e1004503.

39. Farmanbar A, Kneller R, Firouzi S. RNA sequencing identifies clonal structure of T-cell repertoires in patients with adult T-cell leukemia/lymphoma. NPJ Genom Med. 2019;4:10

40. Bolotin DA, Poslavsky S, Mitrophanov I, Shugay M, Mamedov IZ, Putintseva EV, et al. MiXCR: software for comprehensive adaptive immunity profiling. Nat Methods. 2015;12:380-1.

41. Li L, Wu D, Yu Q, Li L, Wu P. Prognostic value of FOXM1 in solid tumors: a systematic review and meta-analysis. Oncotarget. 2017;8:32298-308.

42. Sondka Z, Bamford S, Cole CG, Ward SA, Dunham I, Forbes SA. The COSMIC Cancer Gene Census: describing genetic dysfunction across all human cancers. Nat Rev Cancer. 2018;18:696-705.

43. Klintman J, Appleby N, Stamatopoulos B, Ridout K, Eyre TA, Robbe P, et al. Genomic and transcriptomic correlates of Richter's transformation in Chronic Lymphocytic Leukemia. Blood 2021;137:2800-16.

44. Chen J, Xu-Monette ZY, Deng L, Shen Q, Manyam GC, Martinez-Lopez A, et al. Dysregulated CXCR4 expression promotes lymphoma cell survival and independently predicts disease progression in germinal center B-cell-like diffuse large B-cell lymphoma. Oncotarget. 2015;6:5597-614.

45. Hunter ZR, Xu L, Yang G, Zhou Y, Liu X, Cao Y, et al. The genomic landscape of Waldenstrom macroglobulinemia is characterized by highly recurring MYD88 and WHIM-like CXCR4 mutations, and small somatic deletions associated with B-cell lymphomagenesis. Blood. 2014;123:1637-46.

46. Roccaro AM, Sacco A, Purschke WG, Moschetta M, Buchner K, Maasch C, et al. SDF-1 inhibition targets the bone marrow niche for cancer therapy. Cell Rep. 2014;9:118-28.

47. Cao Y, Hunter ZR, Liu X, Xu L, Yang G, Chen J, et al. The WHIM-like CXCR4(S338X) somatic mutation activates AKT and ERK, and promotes resistance to ibrutinib and other agents used in the treatment of Waldenstrom's Macroglobulinemia. Leukemia. 2015;29:169-76.

48. Dono M, Cerruti G, Zupo S. The CD5+ B-cell. Int J Biochem Cell Biol. 2004;36:2105-11.

49. Anderson KJ, Osvaldsdottir AB, Atzinger $B$, Traustadottir GA, Jensen KN, Larusdottir $A E$, et al. The BLIMP1-EZH2 nexus in a non-Hodgkin lymphoma. Oncogene. 2020;39:5138-51.

50. Morschhauser F, Tilly $\mathrm{H}$, Chaidos A, McKay P, Phillips $T$, Assouline $S$, et al. Tazemetostat for patients with relapsed or refractory follicular lymphoma: an open-label, single-arm, multicentre, phase 2 trial. Lancet Oncol. 2020;21:1433-42.

51. Biajoux V, Natt J, Freitas C, Alouche N, Sacquin A, Hemon P, et al. Efficient plasma cell differentiation and trafficking require Cxcr4 desensitization. Cell Rep. 2016;17:193-205.

52. Alouche N, Bonaud A, Rondeau V, Hussein-Agha R, Nguyen J, Bisio V, et al. Hematological disorder associated Cxcr4-gain-of-function mutation leads to uncontrolled extrafollicular immune response. Blood 2021;137:3050-63.

53. Moriuchi M, Moriuchi H, Margolis DM, Fauci AS. USF/c-Myc enhances, while YinYang 1 suppresses, the promoter activity of CXCR4, a coreceptor for HIV-1 entry. J Immunol. 1999;162:5986-92.

54. Fu Z, Malureanu L, Huang J, Wang W, Li H, van Deursen JM, et al. Plk1-dependent phosphorylation of FoxM1 regulates a transcriptional programme required for mitotic progression. Nat Cell Biol. 2008;10:1076-82.

55. Wang S, Zhang S, Li J, Xu X, Weng Y, Zheng M, et al. CXCL12-induced upregulation of FOXM1 expression promotes human glioblastoma cell invasion. Biochem Biophys Res Commun. 2014;447:1-6.

56. Yao S, Fan LY, Lam EW. The FOXO3-FOXM1 axis: a key cancer drug target and a modulator of cancer drug resistance. Semin Cancer Biol. 2018;50:77-89.

57. Kasahara K, Goto H, Izawa I, Kiyono T, Watanabe N, Elowe S, et al. PI 3-kinasedependent phosphorylation of Plk1-Ser99 promotes association with 14-3-3gamma and is required for metaphase-anaphase transition. Nat Commun. 2013;4:1882.

58. Uddin S, Hussain AR, Ahmed M, Siddiqui K, Al-Dayel F, Bavi P, et al. Overexpression of FoxM1 offers a promising therapeutic target in diffuse large B-cell lymphoma. Haematologica. 2012;97:1092-100.

59. Hassan QN 2nd, Alinari L, Byrd JC. PLK1: a promising and previously unexplored target in double-hit lymphoma. J Clin Investig. 2018;128:5206-8.
60. Sadasivam S, Duan S, DeCaprio JA. The MuvB complex sequentially recruits B-Myb and FoxM1 to promote mitotic gene expression. Genes Dev. 2012;26:474-89.

61. Fischer M, Grossmann P, Padi M, DeCaprio JA. Integration of TP53, DREAM, MMBFOXM1 and RB-E2F target gene analyses identifies cell cycle gene regulatory networks. Nucleic Acids Res. 2016;44:6070-86.

62. Lefebvre C, Rajbhandari P, Alvarez MJ, Bandaru P, Lim WK, Sato M, et al. A human B-cell interactome identifies MYB and FOXM1 as master regulators of proliferation in germinal centers. Mol Syst Biol. 2010;6:377.

63. Subramanian A, Tamayo P, Mootha VK, Mukherjee S, Ebert BL, Gillette MA, et al. Gene set enrichment analysis: a knowledge-based approach for interpreting genome-wide expression profiles. Proc Natl Acad Sci USA. 2005;102:15545-50.

64. Liberzon A, Subramanian A, Pinchback R, Thorvaldsdottir H, Tamayo P, Mesirov JP. Molecular signatures database (MSigDB) 3.0. Bioinformatics. 2011;27:1739-40.

\section{ACKNOWLEDGEMENTS}

Part of the results shown here is based on data generated by the TCGA Research Network: http://cancergenome.nih.gov/. SH is a participant in the BIH-Charité Clinician Scientist Program funded by the Charité - Universitätsmedizin Berlin and the Berlin Institute of Health. RL is a participant in the Doctoral Program in Translational Medicine Program of Technische Universität München.

\section{AUTHOR CONTRIBUTIONS}

Conception and design of the study: $\mathrm{SH}, \mathrm{RL}, \mathrm{UK}$; Acquisition of data and/or analysis and interpretation of data: RL, SH, HCM, NS, IG-M, MW, MS, LZ, KI, AKS, VS, JL, TZ, KS, $\mathrm{RR}$, LQ-M, ME, KB; Drafting of the manuscript: $\mathrm{SH}, \mathrm{RL}$, UK; All authors revised the manuscript for important intellectual content and approved the final version submitted for publication.

\section{FUNDING}

This work was supported by Deutsche Forschungsgemeinschaft (DFG, SFB1335/P3 to UK), Deutsche Krebshilfe (70114425 and 111944 to UK), Stiftung Charite (to UK). The study was further supported by an ANR JCJC grant (ANR-19-CE15-0019-01), and a "Fondation ARC pour la recherche sur le cancer" grant (P JA20181208173) to ME, an ANR PRC grant (ANR-17-CE14-0019) and an INCa grant (PRT-K 2017) to KB. Open Access funding enabled and organized by Projekt DEAL.

\section{COMPETING INTERESTS}

The authors declare no competing interests.

\section{ADDITIONAL INFORMATION}

Supplementary information The online version contains supplementary material available at https://doi.org/10.1038/s41375-021-01376-1.

Correspondence and requests for materials should be addressed to U.K. or S.H.

Reprints and permission information is available at http://www.nature.com/ reprints

Publisher's note Springer Nature remains neutral with regard to jurisdictional claims in published maps and institutional affiliations.

\footnotetext{
Open Access This article is licensed under a Creative Commons

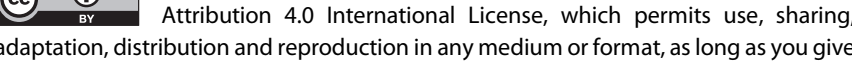
appropriate credit to the original author(s) and the source, provide a link to the Creative Commons license, and indicate if changes were made. The images or other third party material in this article are included in the article's Creative Commons license, unless indicated otherwise in a credit line to the material. If material is not included in the article's Creative Commons license and your intended use is not permitted by statutory regulation or exceeds the permitted use, you will need to obtain permission directly from the copyright holder. To view a copy of this license, visit http://creativecommons. org/licenses/by/4.0/.
}

(c) The Author(s) 2021 